\title{
Traumatic Boutonnière Deformity of the Thumb
}

\section{Deformidad traumática en boutonnière del pulgar}

\author{
Antonio García-jiménez ${ }^{1}$ Bernardo Uran ${ }^{1}$ Javier Ochoa ${ }^{1}$ \\ ${ }^{1}$ Orthopedic Surgery and Traumatology Service, Serveis de Salut \\ Integrats Baix Empordà (SSIBE), Hospital de Palamós, Palamós, \\ Girona, Spain \\ Address for correspondence Antonio García-jiménez, MD, Servicio de \\ Cirugía Ortopédica y Traumatología, Serveis de Salut Integrats Baix \\ Empordà (SSIBE), Hospital de Palamós, Carrer Hospital 36, 17230
}

Rev Iberam Cir Mano 2020;48:133-137.

\begin{abstract}
Keywords

- thumb

- boutonnière

- deformity

- trauma

Resumen

Palabras clave

- pulgar

- boutonnière

- deformidad

- traumatismo

Two Cases Of Boutonnière Deformity After Trauma Are Presented In Patients Without Rheumatic Disease. One Of Them Suffered An Assault and the other a high-energy accident. Both cases were misdiagnosed. The first case presented an injury of the dorsoradial capsule of the metacarpophalangeal (MCP) joint and the second case presented an injury of the adductor pollicis (AP) which is an unusual finding regarding this kind of deformity. The surgical treatment failed up to two times in the first case hile in the second case it failed once.

Se presentan dos casos de deformidad en boutonnière tras traumatismo en pacientes sin antecedentes de enfermedad reumatológica. Uno de ellos había sufrido una agresión, y el otro, un accidente de alta energía. Ambos fueron diagnosticados tardíamente de forma clínica. En el primer caso, se encontró una lesión de la cápsula dorsoradial de la articulación metacarpofalángica (MCF), mientras que en el segundo se encontró una lesión del aductor del pulgar (AP), hallazgo novedoso en este tipo de deformidad. El tratamiento quirúrgico fracasó hasta en dos ocasiones en el primer caso, mientras que en el segundo caso fracasó una vez.
\end{abstract}

\section{Introduction}

The boutonnière deformity of the thumb consists of flexion of the metacarpophalangeal (MCP) joint and extension of the interphalangeal (IP) joint. It is relatively common in rheumatoid arthritis, and it is caused by involvement of the dorsal capsule of the MCP joint secondary to inflammation of the synovial membrane, the hallmark of this disease. ${ }^{1}$ This capsular injury produces ulnar dislocation of the extensor pollicis longus (EPL) tendon; this altered position results in MCP flexion, rather than extension, in addition to IP hyperextension. A similar deformity can occur after traumatic rupture of the dorsoradial capsule, with ulnar dislocation of the EPL tendon, accompanied or not by a lesion of the extensor pollicis brevis (EPB) tendon. These traumatic cases may not present IP joint hyperextension. ${ }^{2-4}$

The present article describes two cases of boutonnière deformity of the thumb secondary to trauma in patients with no rheumatic disease. In addition, a review of the scarce literature about this particular injury is presented, especially regarding its anatomy, diagnosis, and treatment.

\section{Case 1}

A 27-year-old woman with no known history of rheumatological disease or any other relevant medical condition. She was examined at the outpatient traumatology unit due to pain and extension deficit of the MCP and IP joints of the right received

March 23, 2020

accepted

July 27, 2020
DOI https://doi.org/

10.1055/s-0040-1718455. ISSN 1698-8396.
Copyright $\odot 2020$ Thieme Revinter Publicações Ltda, Rio de Janeiro, Brazil
License terms

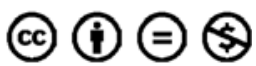




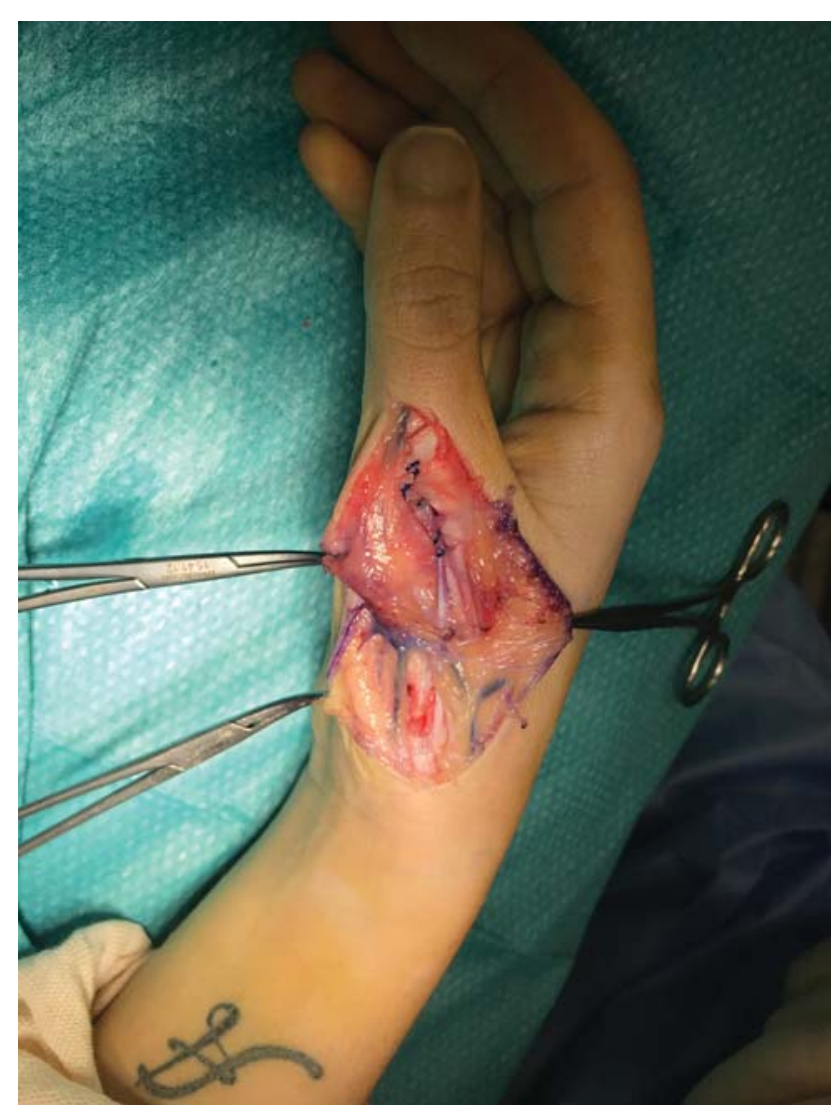

Fig. 1 Intraoperative photograph of patient \#1. Suture of the partial lesion at the extensor pollicis brevis and the dorsoradial capsule to reduce the dislocation at the extensor pollicis longus tendon.

thumb. She reported a traumatic twist of her thumb in an assault two months earlier.

The physical examination revealed $10^{\circ}$ of flexion of the MCP joint and $20^{\circ}$ of flexion of the IP joint, with no active extension capacity. The EPL tendon was palpable on the ulnar side of the finger, and was manually reduced, achieving a slight active extension down to $0^{\circ}$ in both the MCP and IP joints. There was no varus or valgus instability at the MCP joint.

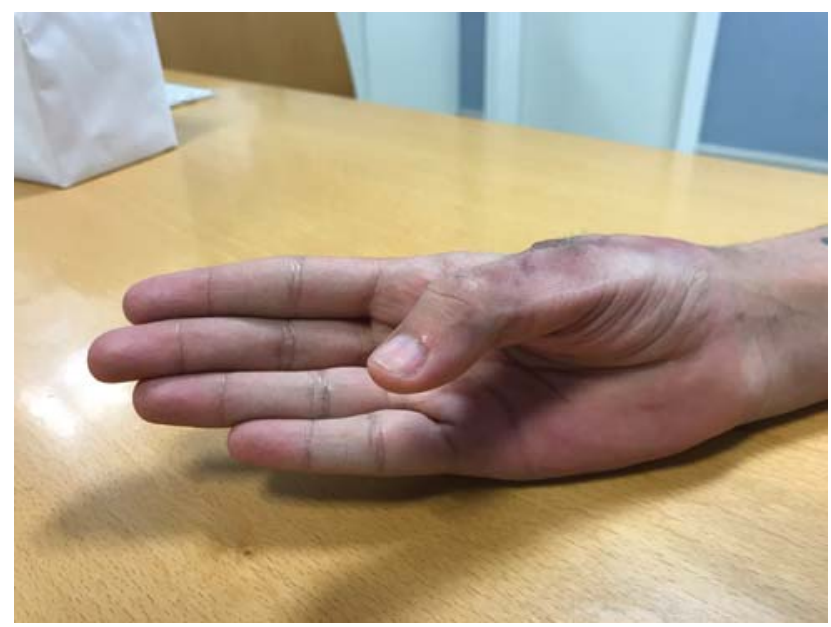

Fig. 2 Clinical photograph of patient \#1, revealing clinical recurrence of the boutonnière deformity with no extension to the interphalangeal joint.
Plain radiographs showed a normal MCP joint, while magnetic resonance imaging (MRI) showed an intact EPL tendon with ulnar subluxation, and disruption of the dorsal capsule in the area of attachment of the EPB; it was not possible to ascertain if this tendon was intact. No injury to the radial or ulnar collateral ligaments of the thumb was observed. Based on these findings, we decided for the surgical treatment of the lesion.

Intraoperatively, the dorsoradial capsule was ruptured, with partial detachment of the EPB tendon at the capsular area and ulnar dislocation of the EPL tendon. The partial EPB lesion was repaired with non-absorbable monofilament polypropylene suture (Prolene, Ethicon, Inc., Bridgewater, NJ, US), whereas the dorsoradial capsule was repaired with absorbable polyglactin suture (Vycril, Ethicon, Inc.) for the reduction of the EPL dislocation (- Figure $\mathbf{1}$ ).

The MCP joint was immobilized for four weeks. After that, the patient started the rehabilitation treatment. Two weeks later, the patient came to the emergency department complaining of acute pain in the operated thumb due to recurrence of the boutonnière deformity (-Figure 2). Another surgery was performed, revealing disruption of the dorsoradial capsule and ulnar dislocation of the EPL tendon, this time with no involvement of the EPB tendon (- Figure 3). A capsular suture was performed with Vycril for the reduction of the EPL dislocation. A different surgical technique was not

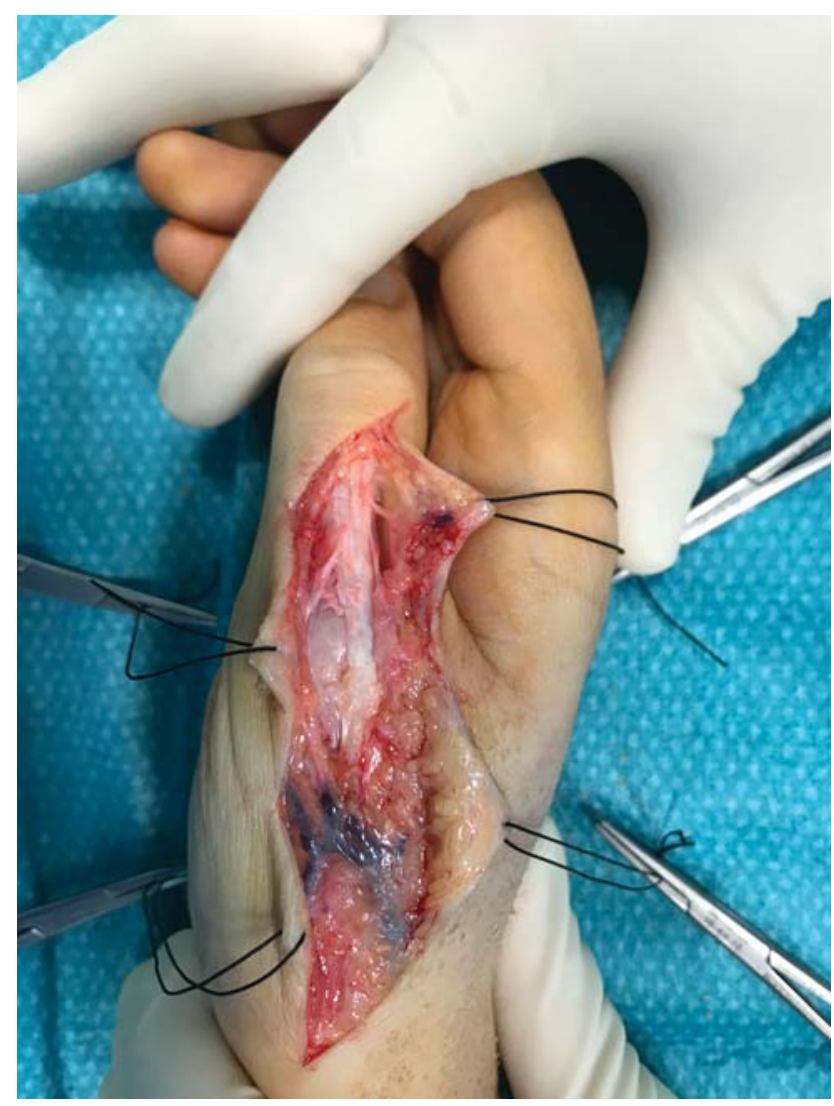

Fig. 3 Intraoperative photograph of patient \#1, revealing recurrence of the boutonnière deformity, disruption of the dorsoradial capsule, and ulnar dislocation of the extensor pollicis longus tendon. 

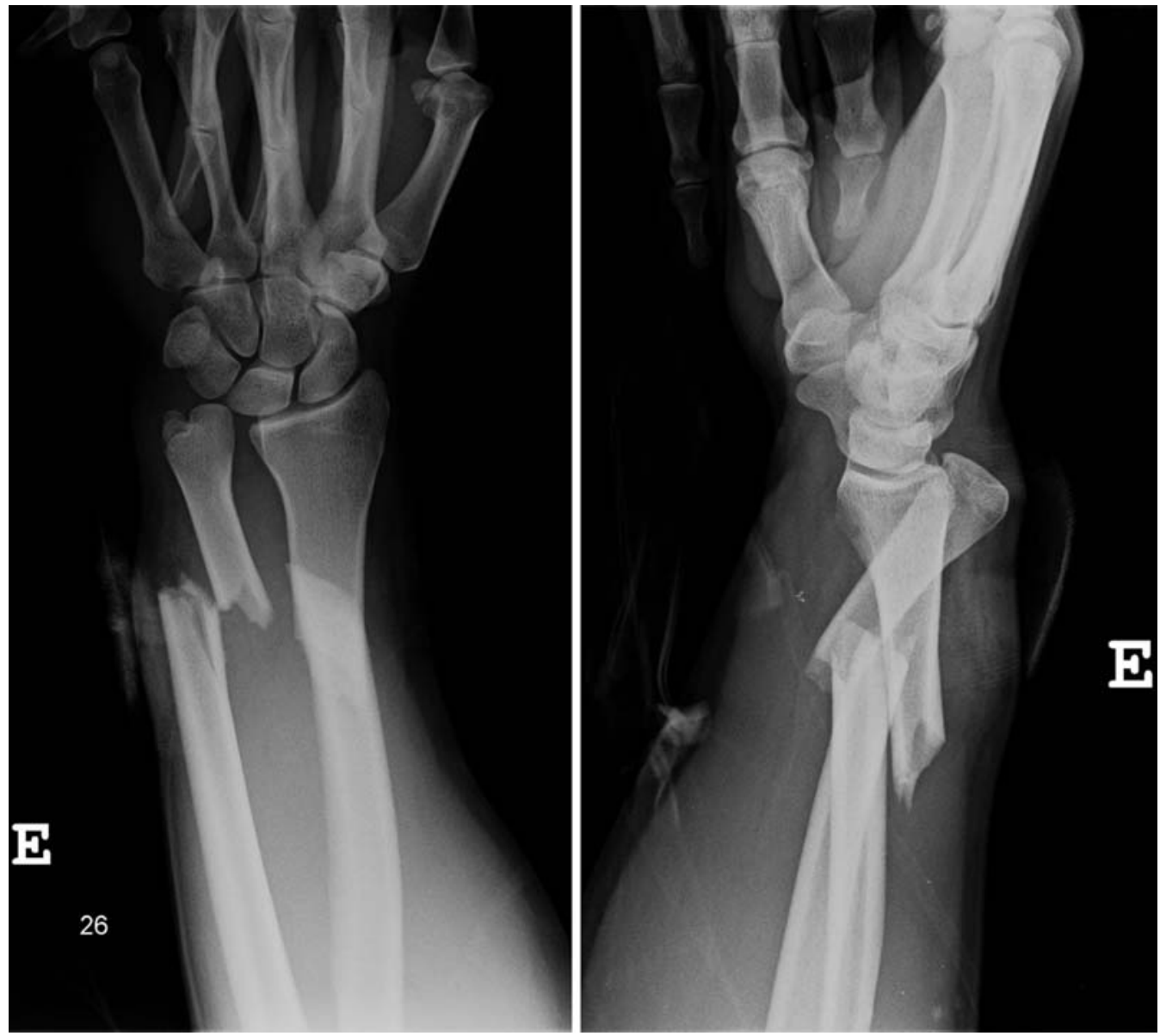

Fig. 4 Plain, posteroanterior and profile radiographs of the left wrist and distal third of the forearm, revealing radial and distal ulnar metaphyseal-diaphyseal fractures, with radial-distal ulnar dislocation.

performed because the quality of the remaining capsule was considered sufficient to repeat the same procedure.

After six weeks of immobilization, the rehabilitation treatment was resumed. The patient was lost to follow-up two months after the second surgery, and she came to the outpatient service 21 months later with a new clinical recurrence. Since the patient prefers an expectant treatment, no surgery will be performed, at least for now.

\section{Case 2}

A 39-year-old man with no known history of rheumatological disease or any other relevant medical condition. He came to our emergency room brought by a conventional ambulance after falling off a motorcycle. He presented radial and distal metaphyseal-diaphyseal ulnar fractures on the left side, with open distal radioulnar dislocation, Gustilo-Anderson grade I ( - Figure 4). Immobilization and prophylactic antibiotic treatment with cefazolin and gentamicin were performed, followed by surgery 12 hours after admission. Open reduction was performed using a double approach and internal fixation of the fracture with low-contact dynamic compression plates (LC-DCP) (-Figure 5).

In an outpatient follow-up visit two weeks after surgery, a thumb extension deficit was observed, but specifics on the physical examination are unknown because it was not performed by the author, and its findings were not recorded at the patient's medical history file. The rehabilitation treatment started and lasted for approximately two months. Since there was no improvement, an ultrasound examination was performed, and revealed no injuries at the extensor apparatus. However, considering the clinical suspicion of eupture of the EPL tendon, surgery was indicated. Intraoperatively, the tendon was intact, but plication was performed due to suspicion of EPL elongation. A splint was placed and kept for 6 weeks, followed by rehabilitation. The patient still presented extension deficit, and he was referred for evaluation at the Hand Unit 15 months after the first surgery, 13 months after the second procedure.

A physical examination revealed a classic boutonnière deformity of the thumb with $40^{\circ}$ of flexion at the MCP joint and $40^{\circ}$ of extension at the IP joint (- Figure 6 ). The MCP joint presented no varus or valgus instability. Since there was no swelling or pain at the MCP region, and the EPL tendon was not palpable at the ulnar area, the patient agreed to an exploratory surgery at the extensor apparatus of the thumb.

The intraoperative assessment revealed a rupture of the dorsal expansion of the adductor pollicis (AP) with significant fibrosis, but no involvement of the EPB tendon, and a radial dislocation of the EPL tendon ( - Figure 7 ). The dorsal expansion remnant of the AP was sutured to the dorsal capsule for the reduction of the EPL. Two Kirschner wires 


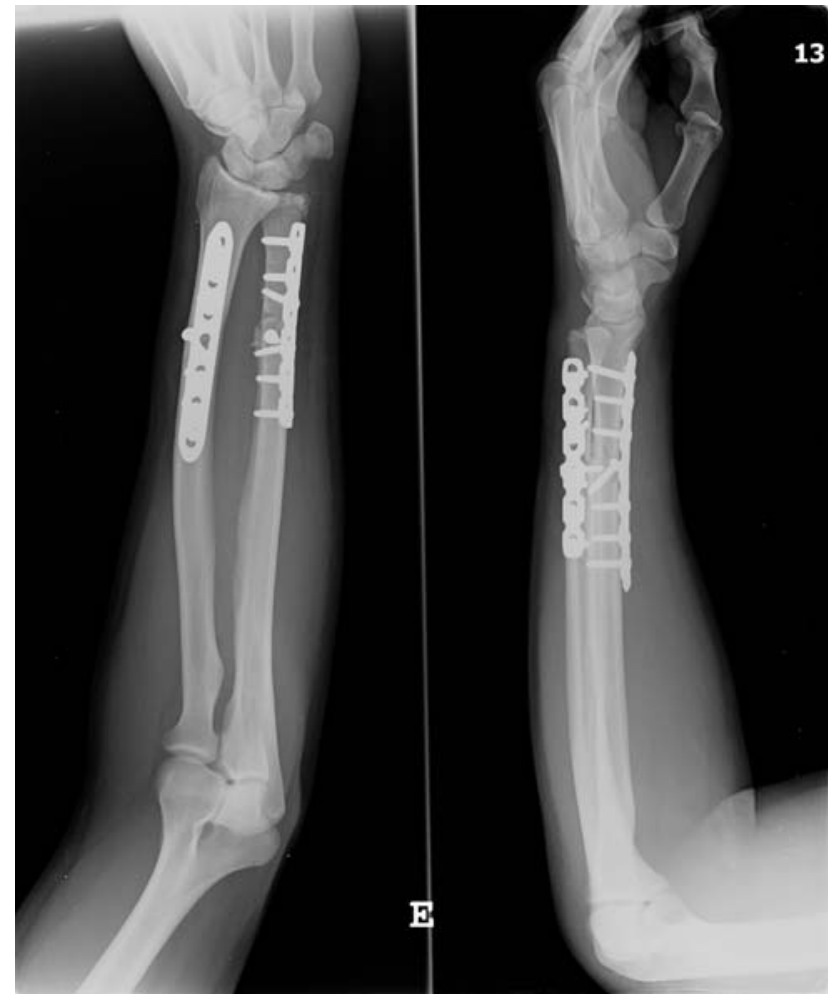

Fig. 5 Plain, posteroanterior and profile radiographs of the forearm. Internal fixation with low-contact dynamic compression plates (LCDCP) of the lesion shown in - Figure 4.

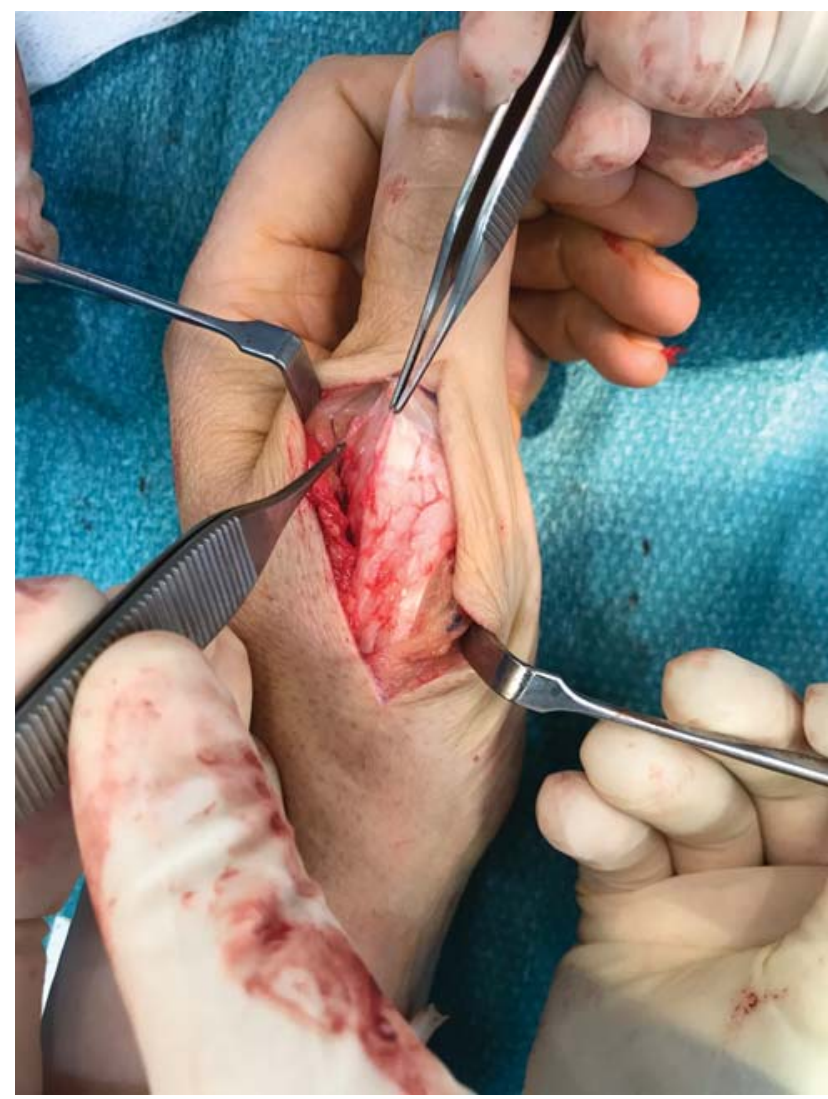

Fig. 7 Intraoperative image of recurrence of the boutonnière deformity with rupture of the adductor. This image also reveals a radial dislocation of the extensor pollicis longus tendon, which was reduced using an Adson forceps.

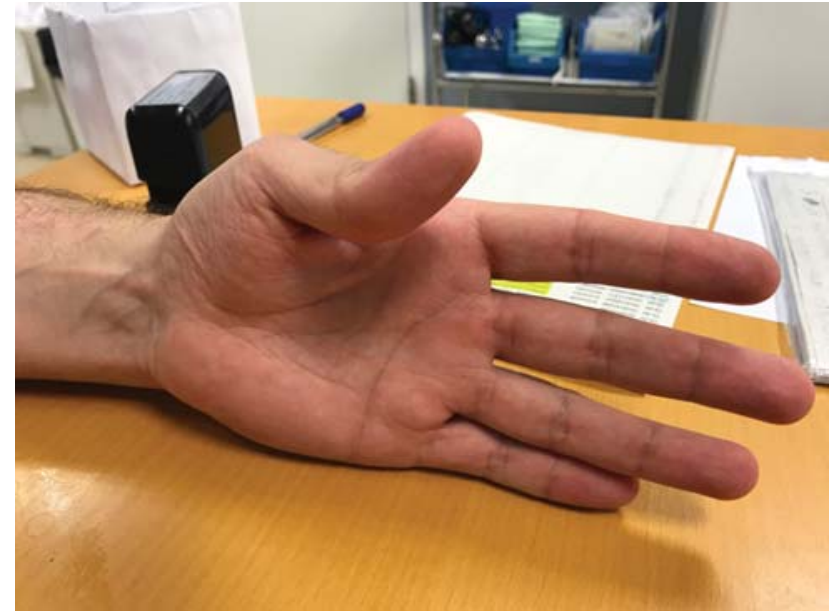

Fig. 6 Clinical photograph of patient \#2, revealing recurrence of the boutonnière deformity with extension to the interphalangeal joint.

transfixed the MCP, and the patient used a cast for 6 weeks. Next, both the cast and the Kirschner wires were removed, and rehabilitation started.

The patient presented progressive clinical recurrence up to 9 months after the last surgery. A surgical revision was offered, but the patient refused it and requested discharge from the outpatient clinic.

\section{Discussion}

Traumatic boutonnière deformity of the thumb is an exceptional injury. There are a number of articles about injuries of the dorsal capsule of the MCP joint, ${ }^{2-6}$ but only two reports specifically mentioned it by name, ${ }^{2,4}$ with a total of seven patients.

The dorsal region of the MCP joint consists of two planes. The deepest plane corresponds to the level of the dorsal capsule. The EPB passes above it until its attachment on the capsule or the proximal phalanx. The most superficial plane corresponds to the dorsal expansions of the AP medially, and the abductor pollicis brevis (APB) laterally. ${ }^{3}$

Traumatic boutonnière deformity of the thumb is a combination of dorsoradial capsule injuries, which may include a complete or partial lesion of the EPB attachment, ${ }^{2-4}$ as seen in the two cases herein presented. The dorsal expansion of the AP drags the EPL tendon towards the ulnar, changing its force vector; as such, the MCP joint is flexed when the patient tries to extend the thumb. The author did not find any previous article, either in Spanish or English, describing that this deformity could result from an AP lesion with radial EPL dislocation, as in the second case.

The diagnosis is clinical, based on the flexion of the MCP joint of the thumb and an inability to extend it. Unlike the boutonnière deformity resulting from rheumatic disease, extension of the IP joint is not required, possibly because the dorsal expansion of the AP joint prevents the proximal migration of the EPL tendon. ${ }^{2}$ For instance, IP involvement was not observed in the first case, but it was present in the second patient. Pressure on the ulnar side of the MCP joint by the examiner can realign the dislocated EPL tendon, 


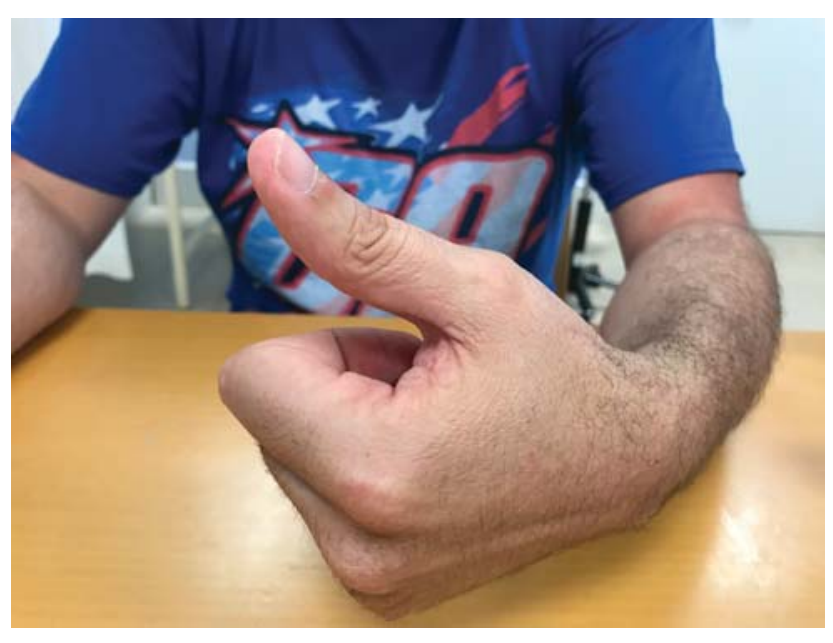

Fig. 8 Clinical photograph of patient \#2. In the boutonnière deformity of the thumb caused by injury of the adductor pollicis, palmar flexion of the wrist can normalize the force vector of the extensor pollicis longus tendon, resulting in metacarpophalangeal extension.

leading to extension of the MCP joint. ${ }^{2}$ When the deformity results from an AP injury, palmar flexion of the wrist can place the EPL tendon over its normal force vector, leading to extension of the MCP joint, as in the second patient (-Figure 8).

An injury diagnosed in its acute form can be treated conservatively with immobilization with thumb extension and satisfactory functional outcomes. ${ }^{2-4}$ For chronic lesions, the treatment can be both conservative and surgical, mostly obtaining good functional outcomes, ${ }^{2-4}$ in contrast to the cases reported in the present article. The surgical techniques used in the literature consist of suture repair or EPB reattachment when it was affected, suture of the capsular lesion and immobilization with Kirschner wires for 4 to 6 weeks. ${ }^{4}$ This is the technique used in both cases. Suturing of the EPL to the EPB to keep it on the dorsum of the MCP joint has also been described. $^{3}$

\section{Conflict of Interests}

The author has no conflict of interests to declare.

\section{References}

1 Nalebuff EA. Diagnosis, classification and management of rheumatoid thumb deformities. Bull Hosp Jt Dis 1968;29(02):119-137

2 Churchill M, Citron N. Isolated subluxation of the extensor pollicis longus tendon. A cause of 'boutonnière' deformity of the thumb. J Hand Surg [Br] 1997;22(06):790-792

3 Le Viet D, Lantieri L. Luxation cubitale du long extenseur du pouce. Etude anatomique et clinique. Ann Chir Main॰••12(03): 173-181(Ann Hand Surg)

4 Cardon LJ, Toh S, Tsubo K. Traumatic boutonniere deformity of the thumb. J Hand Surg [Br] 2000;25(05):505-508

5 Krause JO, Manske PR, Mirly HL, Szerzinski J. Isolated injuries to the dorsoradial capsule of the thumb metacarpophalangeal joint. J Hand Surg Am 1996;21(03):428-433

6 Waitzenegger T, Lantieri L, Le Viet D. Luxation des tendons extenseurs du pouce: étude anatomique, clinique et nouvelle classification. Chir Main 2014;33(04):291-294 